\title{
SUPERMANIFOLDS FROM FEYNMAN GRAPHS
}

\author{
MATILDE MARCOLLI AND ABHIJNAN REJ
}

\begin{abstract}
We generalize the computation of Feynman integrals of log divergent graphs in terms of the Kirchhoff polynomial to the case of graphs with both fermionic and bosonic edges, to which we assign a set of ordinary and Grassmann variables. This procedure gives a computation of the Feynman integrals in terms of a period on a supermanifold, for graphs admitting a basis of the first homology satisfying a condition generalizing the log divergence in this context. The analog in this setting of the graph hypersurfaces is a graph supermanifold given by the divisor of zeros and poles of the Berezinian of a matrix associated to the graph, inside a superprojective space. We introduce a Grothendieck group for supermanifolds and we identify the subgroup generated by the graph supermanifolds. This can be seen as a general procedure to construct interesting classes of supermanifolds with associated periods.
\end{abstract}

\section{Contents}

1. Introduction

1.1. Graph varieties and periods

1.2. Grothendieck ring

2. Supermanifolds and motives

2.1. Supermanifolds

2.2. A Grothendieck group

2.3. Integration on supermanifolds

2.4. Divisors

3. Supermanifolds from graphs

3.1. Feynman's trick and Schwinger parameters 8

3.2. The case of Grassmann variables 10

3.3. Graphs with fermionic legs 12

3.4. Graph supermanifolds 16

3.5. Examples from Feynman graphs 17

3.6. The universality property 18

4. Supermanifolds and mirrors $\quad 19$

References $\quad 20$

\section{INTRODUCTION}

The investigation of the relation between Feynman integrals and motives originates in the work of Broadhurst and Kreimer [9], where it is shown that zeta and multiple zeta values appear systematically in the evaluation of Feynman diagrams. These are very special periods, namely they are believed to arise as periods of mixed Tate motives. An important question in the field then became understanding the a priori reason for the appearance of this special class of motives in quantum field theory. Surprisingly, the work of Belkale and Brosnan [3] revealed a universality result for the varieties associated to Feynman graphs, namely they generate the Grothendieck ring of varieties. This means 
that, as motives, they can be arbitrarily far from the mixed Tate case. The question then moved on to whether the piece of the cohomology of the graph hypersurface complement, which is involved in the evaluation of the Feynman integral as a period, actually happens to be mixed Tate. The recent results of [8], see also [7, analyze this problem in depth in the case of the "wheels with n-spokes" graphs. There are considerable technical difficulties involved in the cohomological calculations, even for relatively uncomplicated graphs, due to the singularities of the graph hypersurfaces and to the fact that generally their complexity grows very rapidly with the combinatorial complexity of the graphs. A different approach to the relation between Feynman integrals and mixed Tate motives was given by Connes-Marcolli in [1], from the point of view of Tannakian categories and motivic Galois groups. This approach originated from the earlier work of Connes-Kreimer [10] where it is shown that the Feynman graphs of a given physical theory form a commutative, non-cocommutative, Hopf algebras. This defines dually an affine group scheme, called the group of diffeographisms of the theory, whose Lie algebra bracket is given by the symmetrized insertion of one graph into another at vertices. The Connes-Kreimer Hopf algebra structure of perturbative renormalization was extended from the case of scalar field theories to the case of QED, and more general gauge theories, by van Suijlekom in [24]. He showed that the Ward identities define a Hopf ideal in the Connes-Kreimer Hopf algebra of Feynman graphs. A related question of motivic lifts of the Connes-Kreimer Hopf algebra is formulated in [7].

The fact that the graph hypersurfaces generate the Grothendieck ring of varieties means that the computation of the Feynman integral in terms of a period on the complement of a graph hypersurface in a projective space gives a general procedure to construct a large class of interesting varieties with associated periods. Our purpose here is to show that this general procedure can be adapted to produce a large class of interesting supermanifolds with associated periods.

In the setting of [8] and 7] one is assuming, from the physical viewpoint, that all edges of the graph are of the same nature, as would be the case in a scalar field theory with Lagrangian

$$
\mathcal{L}(\phi)=\frac{1}{2}(\partial \phi)^{2}-\frac{m^{2}}{2} \phi^{2}-\mathcal{L}_{\text {int }}(\phi) .
$$

However, in more general theories, one has graphs that are constructed out of different types of edges, which correspond to different propagators in the corresponding Feynman rules. We consider the case of theories with fermions, where graphs have both fermionic and bosonic legs. From the mathematical point of view, it is natural to replace the usual construction of the graph hypersurface by a different construction which assigns to the edges either ordinary variables (bosonic) or Grassmann variables (fermionic). This procedure yields a natural way to construct a family of supermanifolds associated to this type of Feynman graphs.

We give a computation of the Feynman integral in terms of a bosonic and a fermionic integration, so that the integral is computed as a period on a supermanifold that is the complement of a divisor in a superprojective space, defined by the set of zeros and poles of the Berezinian of a matrix $\mathcal{M}(t)$ associated to a graph $\Gamma$ and a choice of a basis $B$ for $H_{1}(\Gamma)$. We refer to the divisor defined by this Berezinian as the graph supermanifold $\mathcal{X}_{(\Gamma, B)}$.

As in the case of the ordinary graph hypersurfaces, we are interested in understanding their motiving nature first by looking at their classes in the Grothendieck ring of varieties. To this purpose, we introduce a Grothendieck ring $K_{0}\left(\mathcal{S} \mathcal{V}_{\mathbb{C}}\right)$ of supermanifold and we prove that it is a polynomial ring $K_{0}\left(\mathcal{V}_{\mathbb{C}}\right)[T]$ over the Grothendieck ring of ordinary varieties. We then use this result to prove that the classes of the graph supermanifolds $\mathcal{X}_{(\Gamma, B)}$ generate 
the subring $K_{0}\left(\mathcal{V}_{\mathbb{C}}\right)\left[T^{2}\right]$, where the degree two appears due to a fermion doubling used in the computation of the Feynman integral.

In a different perspective, an interest in supermanifolds and their periods has recently surfaced in the context of mirror symmetry, see [23], 2], [13]. We do not know, at present, whether the classes of supermanifolds considered here and their periods may be of any relevance to that context. We mention some points of contact in $\$ 4$ below.

As the referee pointed out to us, a theory of parametric Feynman integrals for scalar supersymmetric theories was developed in [17. The type of integrals we are considering here is slightly different from those of [17, hence we cannot apply directly the results of that paper. It would be interesting to see what class of graph supermanifolds can be obtained from the parametric integrals of [17].

Acknowledgment. The first author is partially supported by NSF grant DMS0651925. The second author is supported as a Marie Curie Early Stage Researcher at Durham University and by the Clay Mathematical Institute.

1.1. Graph varieties and periods. The evaluation of Feynman integrals in perturbative quantum field theory can be expressed, in the case of logarithmically divergent graphs (which have $n$ loops and $2 n$ edges), in terms of a period in the algebro-geometric sense. This is obtained as the integration over a simplex of an algebraic differential form involving the graph polynomial of the Feynman graph $(c f$. [], [8])

$$
\int_{\sigma} \frac{\Omega}{\Psi_{\Gamma}^{2}}
$$

with $\Psi_{\Gamma}$ the graph polynomial (Kirchhoff polynomial) of the graph $\Gamma, \sigma$ the simplex in $\mathbb{P}^{2 n-1}$, and

$$
\Omega=\sum_{i=1}^{2 n}(-1)^{i} x_{i} d x_{1} \cdots \widehat{d x_{i}} \cdots d x_{n} .
$$

The logarithmically divergent case is the one where periods are defined independently of a renormalization procedure. In the more general case, the problem arises from the fact that the integrand acquires poles along exceptional divisors in the blowup along faces of the simplex (see [8, [7]).

In the following, given a graph $\Gamma$ we denote by $\Psi_{\Gamma}$ the graph polynomial

$$
\Psi_{\Gamma}(x)=\sum_{T \subset \Gamma} \prod_{e \notin T} x_{e}
$$

where the sum is over all the spanning trees $T$ of $\Gamma$ and the product is over edges not belonging to $T$. These give homogeneous polynomials in the variables $x=\left(x_{e}\right)=$ $\left(x_{1}, \cdots, x_{\# E(\Gamma)}\right)$ associated to the edges of $\Gamma$, where each variable appears of degree at most one in each monomial. They define hypersurfaces

$$
X_{\Gamma}=\left\{x=\left(x_{e}\right) \in \mathbb{P}^{\# E(\Gamma)-1} \mid \Psi_{\Gamma}(x)=0\right\} .
$$

These are typically singular hypersurfaces.

In the case of the log divergent graphs considered in [8], the motive involved in the evaluation of the Feynman integral as a period is of the form

$$
H^{2 n-1}\left(P \backslash Y_{\Gamma}, \Sigma \backslash\left(\Sigma \cap Y_{\Gamma}\right)\right),
$$

where $n$ is the number of loops, $P \rightarrow \mathbb{P}^{2 n-1}$ is a blowup along linear spaces, $Y_{\Gamma}$ is the strict transform of $X_{\Gamma}$ and $\Sigma$ is the total inverse image of the coordinate simplex of $\mathbb{P}^{2 n-1}$.

The recent results of Bergbauer-Rej [4 provide an explicit combinatorial formula for the graph polynomial under insertion of one graph into another. 
1.2. Grothendieck ring. Recall that the Grothendieck ring $K_{0}\left(\mathcal{V}_{K}\right)$ of varieties is generated by quasi-projective varieties over a field $K$ with the relation

$$
[X]=[Y]+[X \backslash Y]
$$

for $Y \subset X$ a closed subvariety. It is made into a ring by the product of varieties.

Based on computer experiments, which showed that many graph hypersurfaces satisfy the condition

$$
\# X_{\Gamma}\left(\mathbb{F}_{q}\right)=P_{\Gamma}(q)
$$

for some polynomial $P_{\Gamma}$, Kontsevich conjectured that the $X_{\Gamma}$ would be always mixed Tate. The main result of Belkale-Brosnan [3] disproved the conjecture by showing that the classes $\left[X_{\Gamma}\right]$ are very general. In fact, they span the Grothendieck ring of varieties, which means that the $X_{\Gamma}$ can be quite arbitrary as motives. As discussed in [8] and [7, it is especially interesting to construct explicit stratifications of the graph hypersurfaces and try to identify which strata are likely to be non-mixed-Tate.

In the Grothendieck ring $K_{0}\left(\mathcal{V}_{\mathbb{C}}\right)$ the class $\left[\mathbb{A}^{1}\right]=[\mathbb{C}]$ is often denoted by $\mathbb{L}$ and is the class of the Lefschetz motive, with $\left[\mathbb{P}^{1}\right]=1+\mathbb{L}$ and $1=[p t]=\left[\mathbb{A}^{0}\right]$.

There are two opposite ways to deal with the Lefschetz motive $\mathbb{L}$. If, as in the theory of motives, one formally inverts $\mathbb{L}$, one enriches in this way the Grothendieck ring of varieties by the Tate motives $\mathbb{L}^{n}, n \in \mathbb{Z}$. In the theory of motives, one usually denotes $\mathbb{Q}(1)$ the formal inverse of the Lefschetz motive, with $\mathbb{Q}(n)=\mathbb{Q}(1)^{\otimes n}$. The category of pure (respectively, mixed) Tate motives is the subcategory of the abelian (respectively, triangulated) category of motives generated by the $\mathbb{Q}(n)$.

If, instead, one maps the Lefschetz motive $\mathbb{L}$ to zero, one obtains the semigroup ring of stable birational equivalence classes of varieties, by the result of [14, which we briefly recall. Two irreducible varieties $X$ and $Y$ are said to be stably birationally equivalent if $X \times \mathbb{P}^{n}$ is birational to $Y \times \mathbb{P}^{m}$ for some $n, m \geq 0$. It is proved in [14], that there is a ring isomorphism

$$
K_{0}\left[\mathcal{V}_{\mathbb{C}}\right] / I \cong \mathbb{Z}[S B],
$$

where $S B$ is the semigroup of stable birational classes of varieties with the product induced by the product of varieties, $\mathbb{Z}[S B]$ is the associated semigroup ring, and $I \subset K_{0}\left[\mathcal{V}_{\mathbb{C}}\right]$ is the ideal generated by the class $\left[\mathbb{A}^{1}\right]$ of the affine line. The result of [14] essentially depends on the Abramovich-Karu-Matsuki-Wlodarczyk factorization theorem [1], which shows that any rational birational map of smooth complete varieties decomposes as a sequence of blowups and blowdowns, and on Hironaka's resolution of singularities.

\section{Supermanifolds And motives}

2.1. Supermanifolds. We recall here a few basic facts of supergeometry that we need in the following. The standard reference for the theory of supermanifolds is Manin's [16].

By a complex supermanifold one understands a datum $\mathcal{X}=(X, \mathcal{A})$ with the following properties: $\mathcal{A}$ is a sheaf of supercommutative rings on $X ;\left(X, \mathcal{O}_{X}\right)$ is a complex manifold, where $\mathcal{O}_{X}=\mathcal{A} / \mathcal{N}$, with $\mathcal{N}$ the ideal of nilpotents in $\mathcal{A}$; the quotient $\mathcal{E}=\mathcal{N} / \mathcal{N}^{2}$ is locally free over $\mathcal{O}_{X}$ and $\mathcal{A}$ is locally isomorphic to the exterior algebra $\Lambda_{\mathcal{O}_{X}}(\mathcal{E})$, where the grading is the $\mathbb{Z}_{2}$-grading by odd/even degrees. The supermanifold is split if the isomorphism $\mathcal{A} \cong \Lambda_{\mathcal{O}_{X}}(\mathcal{E})$ is global.

Example 2.1. Projective superspace. The complex projective superspace $\mathbb{P}^{n \mid m}$ is the supermanifold $(X, \mathcal{A})$ with $X=\mathbb{P}^{n}$ the usual complex projective space and

$$
\mathcal{A}=\Lambda^{\bullet}\left(\mathbb{C}^{m} \otimes_{\mathbb{C}} \mathcal{O}(-1)\right),
$$

with the exterior powers $\Lambda^{\bullet}$ graded by odd/even degree. It is a split supermanifold. 
A morphism $F: \mathcal{X}_{1} \rightarrow \mathcal{X}_{2}$ of supermanifolds $\mathcal{X}_{i}=\left(X_{i}, \mathcal{A}_{i}\right), i=1,2$, consists of a pair $F=\left(f, f^{\#}\right)$ of a morphism of the underlying complex manifolds $f: X_{1} \rightarrow X_{2}$ together with a morphism $f^{\#}: \mathcal{A}_{2} \rightarrow f_{*} \mathcal{A}_{1}$ of sheaves of supercommutative rings with the property that at each point $x \in X_{1}$ the induced morphism $f_{x}^{\#}:\left(\mathcal{A}_{2}\right)_{f(x)} \rightarrow\left(\mathcal{A}_{1}\right)_{x}$ satisfies $f_{x}^{\#}\left(\mathfrak{m}_{f(x)}\right) \subset \mathfrak{m}_{x}$, on the maximal ideals of germs of sections vanishing at the point $(c f$. [16], §4.1).

In particular, an embedding of complex supermanifolds is a morphism $F=\left(f, f^{\#}\right)$ as above, with the property that $f: X_{1} \hookrightarrow X_{2}$ is an embedding and $f^{\#}: \mathcal{A}_{2} \rightarrow f_{*} \mathcal{A}_{1}$ is surjective. As in ordinary geometry, we define the ideal sheaf of $\mathcal{X}_{1}$ to be the kernel

$$
\mathcal{I}_{\mathcal{X}_{1}}:=\operatorname{Ker}\left(f^{\#}: \mathcal{A}_{2} \rightarrow f_{*} \mathcal{A}_{1}\right) \text {. }
$$

An equivalent characterization of an embedding of supermanifold is given as follows. If we denote by $E_{i}$, for $i=1,2$ the holomorphic vector bundles on $X_{i}$ such that $\mathcal{O}\left(E_{i}\right)=\mathcal{E}_{i}=$ $\mathcal{N}_{i} / \mathcal{N}_{i}^{2}$, with the notation as above, then an embedding $F: \mathcal{X}_{1} \hookrightarrow \mathcal{X}_{2}$ is an embedding $f: X_{1} \hookrightarrow X_{2}$ such that the induced morphism of vector bundles $f^{*}: E_{2} \rightarrow E_{1}$ is surjective (cf. [15]). Thus, we say that $\mathcal{Y}=(Y, \mathcal{B})$ is a closed sub-supermanifold of $\mathcal{X}=(X, \mathcal{A})$ when there exists a closed embedding $Y \subset X$ and the pullback of $E_{\mathcal{A}}$ under this embedding surjects to $E_{\mathcal{B}}$.

An open submanifold $\mathcal{U}=(U, \mathcal{B}) \hookrightarrow \mathcal{X}=(X, \mathcal{A})$ is given by an open embedding $U \hookrightarrow X$ of the underlying complex manifolds and an isomorphism of sheaves $\left.\mathcal{A}\right|_{U} \cong \mathcal{B}$. When $\mathcal{Y} \subset \mathcal{X}$ is a closed embedding and $U=X \backslash Y$, the ideal sheaf of $\mathcal{Y}$ satisfies $\left.\mathcal{I}\right|_{U}=\left.\mathcal{A}\right|_{U}$.

A subvariety in superprojective space is a supermanifold

$$
\mathcal{X}=\left(X \subset \mathbb{P}^{n},\left.\left(\Lambda^{\bullet}\left(\mathbb{C}^{m} \otimes_{\mathbb{C}} \mathcal{O}(-1)\right) / \mathcal{I}\right)\right|_{X}\right),
$$

where $\mathcal{I}=\mathcal{I}_{\mathcal{X}}$ is an ideal generated by finitely many homogeneous polynomials of given $\mathbb{Z} / 2$-parity. In other words, if we denote by $\left(x_{0}, \ldots, x_{n}, \theta_{1}, \ldots, \theta_{m}\right)$ the bosonic and fermionic coordinates of $\mathbb{P}^{n \mid m}$, then a projective subvariety can be obtained by assigning a number of equations of the form

$$
\Psi^{\text {ev/odd }}\left(x_{0}, \ldots, x_{n}, \theta_{1}, \ldots, \theta_{m}\right)=\sum_{i_{1}<\cdots<i_{k}} P_{i_{1}, \ldots, i_{k}}\left(x_{0}, \ldots, x_{n}\right) \theta_{i_{1}} \cdots \theta_{i_{k}}=0,
$$

where the $P_{i_{1}, \ldots, i_{k}}\left(x_{0}, \ldots, x_{n}\right)$ are homogeneous polynomials in the bosonic variables.

Notice that there are strong constraints in supergeometry on realizing supermanifolds as submanifolds of superprojective space. For instance, Penkov and Skornyakov [19] showed that super Grassmannians in general do not embed in superprojective space, cf. [16]. The result of LeBrun, Poon, and Wells [15] shows that a supermanifold $\mathcal{X}=(X, \mathcal{A})$ with compact $X$ can be embedded in some superprojective space $\mathbb{P}^{n \mid m}$ if and only if it has a positive rank-one sheaf of $\mathcal{A}$-modules.

Notice that, in the above, we have been working with complex projective superspace and complex subvarieties. However, it is possible to consider supergeometry in an arithmetic context, as shown in 22], so that it makes sense to investigate extensions of motivic notions to the supergeometry setting. In the present paper we limit our investigation of motivic aspects of supermanifolds to the analysis of their classes in a suitable Grothendieck ring.

2.2. A Grothendieck group. We begin by discussing the Grothendieck group of varieties in the supergeometry context and its relation to the Grothendieck group of ordinary varieties.

We first recall the following notation from 12$] \S I I .2 .3$. Given a locally closed subset $Y \subset X$ and a sheaf $\mathcal{A}$ on $X$, there exists a unique sheaf $\mathcal{A}_{Y}$ with the property that

$$
\left.\mathcal{A}_{Y}\right|_{Y}=\left.\mathcal{A}\right|_{Y} \quad \text { and }\left.\quad \mathcal{A}_{Y}\right|_{X \backslash Y}=0 .
$$


In the case where $Y$ is closed, this satisfies $\mathcal{A}_{Y}=i_{*}\left(\left.\mathcal{A}\right|_{Y}\right)$ where $i: Y \hookrightarrow X$ is the inclusion, and when $Y$ is open it satisfies $\mathcal{A}_{Y}=\operatorname{Ker}\left(\mathcal{A} \rightarrow i_{*}\left(\left.\mathcal{A}\right|_{X \backslash Y}\right)\right)$.

Definition 2.2. Let $\mathcal{S} \mathcal{V}_{\mathbb{C}}$ be the category of complex supermanifolds with morphisms defined as above. Let $K_{0}\left(\mathcal{S} \mathcal{V}_{\mathbb{C}}\right)$ denote the free abelian group generated by the isomorphism classes of objects $\mathcal{X} \in \mathcal{S} \mathcal{V}_{\mathbb{C}}$ subject to the following relations. Let $F: \mathcal{Y} \hookrightarrow \mathcal{X}$ be a closed embedding of supermanifolds. Then

$$
[\mathcal{X}]=[\mathcal{Y}]+[\mathcal{X} \backslash \mathcal{Y}]
$$

where $\mathcal{X} \backslash \mathcal{Y}$ is the supermanifold

$$
\mathcal{X} \backslash \mathcal{Y}=\left(X \backslash Y,\left.\mathcal{A}_{X}\right|_{X \backslash Y}\right) .
$$

In particular, in the case where $\mathcal{A}=\mathcal{O}_{X}$ is the structure sheaf of $X$, the relation (2.5) reduces to the usual relation

$$
[X]=[Y]+[X \backslash Y] .
$$

in the Grothendieck group of ordinary varieties, for a closed embedding $Y \subset X$.

Lemma 2.3. All supermanifolds decompose in $K_{0}\left(\mathcal{S} \mathcal{V}_{\mathbb{C}}\right)$ as a finite combination of split supermanifolds, and in fact of supermanifolds where the vector bundle $E$ with $\mathcal{O}(E)=\mathcal{E}=$ $\mathcal{N} / \mathcal{N}^{2}$ is trivial.

Proof. This is a consequence of the dévissage of coherent sheaves. Namely, for any coherent sheaf $\mathcal{A}$ over a Noetherian reduced irreducible scheme there exists a dense open set $U$ such that such that $\left.\mathcal{A}\right|_{U}$ is free. The relation (2.5) then ensures that, given a pair $\mathcal{X}=(X, \mathcal{A})$ and the sequence of sheaves

$$
0 \rightarrow i_{!}\left(\left.\mathcal{A}\right|_{U}\right) \rightarrow \mathcal{A} \rightarrow j_{*}\left(\left.\mathcal{A}\right|_{Y}\right) \rightarrow 0,
$$

associated to the open embedding $U \subset X$ with complement $Y=X \backslash U$, the class $[X, \mathcal{A}]$ satisfies

$$
[X, \mathcal{A}]=\left[U,\left.\mathcal{A}_{U}\right|_{U}\right]+\left[Y,\left.\mathcal{A}_{Y}\right|_{Y}\right] .
$$

The sheaf $\mathcal{A}_{Y}$ on $X$, which has support $Y$, has a chain of subsheaves $\mathcal{A}_{Y} \supset \mathcal{A}_{1} \supset \cdots \supset$ $\mathcal{A}_{k}=0$ such that each quotient $\mathcal{A}_{i} / \mathcal{A}_{i+1}$ is coherent on $Y$. Thus, one can find a stratification where on each open stratum the supermanifold is split and with trivial vector bundle. The supermanifold $\mathcal{X}=(X, \mathcal{A})$ decomposes as a sum of the corresponding classes in the Grothendieck group.

The fact that the vector bundle that constitutes the fermionic part of a supermanifold is trivial when seen in the Grothendieck group is the analog for supermanifolds of the fact that any projective bundle is equivalent to a product in the Grothendieck group of ordinary varieties.

It follows from Lemma 2.3 above that the product makes $K_{0}\left(\mathcal{S} \mathcal{V}_{\mathbb{C}}\right)$ into a ring with

$$
[\mathcal{X}][\mathcal{Y}]=[\mathcal{X} \times \mathcal{Y}] .
$$

In fact, we have the following more precise description of $K_{0}\left(\mathcal{S} \mathcal{V}_{\mathbb{C}}\right)$ in terms of the Grothendieck ring of ordinary varieties.

Corollary 2.4. The Grothendieck ring $K_{0}\left(\mathcal{S} \mathcal{V}_{\mathbb{C}}\right)$ of supervarieties is a polynomial ring over the Grothendieck ring of ordinary varieties of the form

$$
K_{0}\left(\mathcal{S} \mathcal{V}_{\mathbb{C}}\right)=K_{0}\left(\mathcal{V}_{\mathbb{C}}\right)[T],
$$

where $T=\left[\mathbb{A}^{0 \mid 1}\right]$ is the class of the affine superspace of dimension $(0,1)$. 
It then follows that the relation (1.7) between the Grothendieck ring and the semigroup ring of stable birational equivalence classes extends to this context.

Notice that, in the supermanifold case, there are now two different types of Lefschetz motives, namely the bosonic one $\mathbb{L}_{b}=\left[\mathbb{A}^{1 \mid 0}\right]$ and the fermionic one $\mathbb{L}_{f}=\left[\mathbb{A}^{0 \mid 1}\right]$. By analogy to what happens in motivic integration and in the theory of motives, we may want to localize at the Lefschetz motives, i.e. invert both $\mathbb{L}_{b}$ and $\mathbb{L}_{f}$. That is, according to Corollary 2.4, we consider the field of fractions of $K_{0}\left(\mathcal{V}_{\mathbb{C}}\right)\left[\mathbb{L}_{b}^{-1}\right]=\mathcal{S}^{-1} K_{0}\left(\mathcal{V}_{\mathbb{C}}\right)$, with respect to the multiplicative semigroup $\mathcal{S}=\left\{1, \mathbb{L}_{b}, \mathbb{L}_{b}^{2}, \ldots\right\}$ and then the ring of Laurent polynomials

$$
\mathcal{S}^{-1} K_{0}\left(\mathcal{V}_{\mathbb{C}}\right)\left[\mathbb{L}_{f}, \mathbb{L}_{f}^{-1}\right]=K_{0}\left(\mathcal{V}_{\mathbb{C}}\right)\left[\mathbb{L}_{b}^{-1}, \mathbb{L}_{f}, \mathbb{L}_{f}^{-1}\right]
$$

This suggests extensions of motivic integration to the context of supermanifolds, but we will not pursue this line of thought further in the present paper.

There is also a natural extension to supermanifolds of the usual notion of birational equivalence. We say that two supermanifolds $\mathcal{X}=(X, \mathcal{A})$ and $(Y, \mathcal{B})$ are birational if there exist open dense embeddings of supermanifolds $\mathcal{U} \subset \mathcal{X}$ and $\mathcal{V} \subset \mathcal{Y}$ and an isomorphism $\mathcal{U} \cong \mathcal{V}$. Similarly, one can give a notion analogous to that of stable birational equivalence by requiring that there are superprojective spaces $\mathbb{P}^{n \mid m}$ and $\mathbb{P}^{r \mid s}$ such that $\mathcal{X} \times \mathbb{P}^{n \mid m}$ and $\mathcal{Y} \times \mathbb{P}^{r \mid s}$ are birational. One then finds the following. We denote by $\mathbb{Z}[S S B]$ the semigroup ring of stable birational equivalence classes of supermanifolds.

Corollary 2.5. There is a surjective ring homomorphism $K_{0}\left(\mathcal{S} \mathcal{V}_{\mathbb{C}}\right) \rightarrow \mathbb{Z}[S S B]$, which induces an isomorphism

$$
K_{0}\left(\mathcal{S} \mathcal{V}_{\mathbb{C}}\right) / I \cong \mathbb{Z}[S S B]
$$

where $I$ is the ideal generated by the classes $\left[\mathbb{A}^{1 \mid 0}\right]$ and $\left[\mathbb{A}^{0 \mid 1}\right]$.

The formal inverses of $\mathbb{L}_{f}$ and $\mathbb{L}_{b}$ correspond to two types of Tate objects for motives of supermanifold, respectively fermionic and bosonic Tate motives. We see from Corollary 2.4 and (2.9) that the fermionic part of the supermanifolds only contribution to the class in the Grothendieck ring is always of this fermonic Tate type, while only the bosonic part can provide non-Tate contributions.

2.3. Integration on supermanifolds. The analog of the determinant in supergeometry is given by the Berezinian. This is defined in the following way. Suppose given a matrix $\mathcal{M}$ of the form

$$
\mathcal{M}=\left(\begin{array}{ll}
M_{11} & M_{12} \\
M_{21} & M_{22}
\end{array}\right)
$$

where the $M_{11}$ and $M_{22}$ are square matrices with entries of order zero and the $M_{12}$ and $M_{21}$ have elements of order one. Then the Berezinian of $\mathcal{M}$ is the expression

$$
\operatorname{Ber}(\mathcal{M}):=\frac{\operatorname{det}\left(M_{11}-M_{12} M_{22}^{-1} M_{21}\right)}{\operatorname{det}\left(M_{22}\right)} .
$$

It satisfies $\operatorname{Ber}(\mathcal{M N})=\operatorname{Ber}(\mathcal{M}) \operatorname{Ber}(\mathcal{N})$.

It is shown in [5] that Grassmann integration satisfies a change of variable formula where the Jacobian of the coordinate change is given by the Berezinian $\operatorname{Ber}(J)$ with $J$ the matrix $J=\frac{\partial X_{\alpha}}{\partial Y_{\beta}}$ with $X_{\alpha}=\left(x_{i}, \xi_{r}\right)$ and $Y_{\beta}=\left(y_{j}, \eta_{s}\right)$. We explain in 93 below how to use this to replace expressions of the form (1.2) for Feynman integrals, with similar expressions involving a Berezinian and a Grassmann integration over a supermanifold. 
2.4. Divisors. There is a well developed theory of divisors on supermanifolds, originating from [21]. A Cartier divisor on a supermanifold $\mathcal{X}$ of dimension $(n \mid m)$ is defined by a collection of even meromorphic functions $\phi_{i}$ defined on an open covering $\mathcal{U}_{i} \hookrightarrow \mathcal{X}$, with $\phi_{i} \phi_{j}^{-1}$ a holomorphic function on $\mathcal{U}_{i} \cap \mathcal{U}_{j}$ nowhere vanishing on the underlying $U_{i} \cap U_{j}$. Classes of divisors correspond to equivalence classes of line bundles and can be described in terms of integer linear combinations of $(n-1 \mid m)$-dimensional subvarieties $\mathcal{Y} \subset \mathcal{X}$.

\section{SUPERMANIFOLDS FROM GRAPHS}

3.1. Feynman's trick and Schwinger parameters. We begin by describing a simple generalization of the well known "Feynman trick",

$$
\frac{1}{a b}=\int_{0}^{1} \frac{1}{(x a+(1-x) b)^{2}} d x
$$

which will be useful in the following. The results recalled here are well known in the physics literature (see e.g. [6] $\S 8$ and $\S 18$ ), but we give a brief and self contained treatment here for the reader's convenience. A similar derivation from a more algebro-geometric viewpoint can be found in 8 .

Lemma 3.1. Let $\Sigma_{n}$ denote the $n$-dimensional simplex

$$
\Sigma_{n}=\left\{\left(t_{1}, \ldots, t_{n}\right) \in\left(\mathbb{R}_{+}^{*}\right)^{n} \mid \sum_{i=1}^{n} t_{i} \leq 1\right\} .
$$

Let $d v_{\Sigma_{n}}=d t_{1} \cdots d t_{n-1}$ be the volume form on $\Sigma_{n}$ induced by the standard Euclidean metric in $\mathbb{R}^{n}$. Then, for given nonzero parameters $q_{i}$, for $i=1, \ldots, n$, the following identity holds:

$$
\frac{1}{q_{1} \ldots q_{n}}=(n-1) ! \int_{\Sigma_{n-1}} \frac{1}{\left(t_{1} q_{1}+\cdots+t_{n} q_{n}\right)^{n}} d v_{\Sigma_{n}}
$$

where $t_{n}=1-\sum_{i=1}^{n-1} t_{i}$.

Proof. The following identity holds:

$$
\frac{1}{q_{1}^{k_{1}} \cdots q_{n}^{k_{n}}}=\frac{1}{\Gamma\left(k_{1}\right) \cdots \Gamma\left(k_{n}\right)} \int_{0}^{\infty} \cdots \int_{0}^{\infty} e^{-\left(s_{1} q_{1}+\cdots+s_{n} q_{n}\right)} s_{1}^{k_{1}-1} \cdots s_{n}^{k_{n}-1} d s_{1} \cdots d s_{n} .
$$

The $s_{i}$ are usually called Schwinger parameters in the physics literature. We then perform a change of variables, by setting $S=\sum_{i=1}^{n} s_{i}$ and $s_{i}=S t_{i}$, with $t_{i} \in[0,1]$ with $\sum_{i=1}^{n} t_{i}=1$. Thus, we rewrite (3.3) in the form

$$
\frac{1}{q_{1}^{k_{1}} \cdots q_{n}^{k_{n}}}=\frac{\Gamma\left(k_{1}+\cdots+k_{n}\right)}{\Gamma\left(k_{1}\right) \cdots \Gamma\left(k_{n}\right)} \int_{0}^{1} \cdots \int_{0}^{1} \frac{t_{1}^{k_{1}-1} \cdots t_{n}^{k_{n}-1} \delta\left(1-\sum_{i=1}^{n} t_{i}\right)}{\left(t_{1} q_{1}+\cdots+t_{n} q_{n}\right)^{k_{1}+\cdots+k_{n}}} d t_{1} \cdots d t_{n}
$$

The result (3.2) then follows as a particular case of this more general identity, with $k_{i}=1$ for $i=1, \ldots, n$ and $\Gamma(n)=(n-1)$ !.

One can also give an inductive proof of (3.2) by Stokes theorem, which avoids introducing any transcendental functions, but the argument we recalled here is standard and it suffices for our purposes.

The Feynman trick is then related to the graph polynomial $\Psi_{\Gamma}$ in the following way (see again [6], $\S 18$ and [18]). Suppose given a graph $\Gamma$. Let $n=\# E(\Gamma)$ be the number of 
edges of $\Gamma$ and let $\ell=b_{1}(\Gamma)$ be the number of loops, i.e. the rank of $H^{1}(\Gamma, \mathbb{Z})$. Suppose chosen a set of generators $\left\{l_{1}, \ldots, l_{\ell}\right\}$ of $H^{1}(\Gamma, \mathbb{Z})$. We then define the $n \times \ell$-matrix $\eta_{i k}$ as

$$
\eta_{i k}=\left\{\begin{aligned}
+1 & \text { edge } e_{i} \in \text { loop } l_{k}, \text { same orientation } \\
-1 & \text { edge } e_{i} \in \text { loop } l_{k}, \text { reverse orientation } \\
0 & \text { otherwise. }
\end{aligned}\right.
$$

Also let $M_{\Gamma}$ be the $\ell \times \ell$ real symmetric matrix

$$
\left(M_{\Gamma}\right)_{k r}(t)=\sum_{i=0}^{n} t_{i} \eta_{i k} \eta_{i r},
$$

for $t=\left(t_{0}, \ldots, t_{n-1}\right) \in \Sigma_{n}$ and $t_{n}=1-\sum_{i} t_{i}$. Let $s_{k}, k=1, \ldots, \ell$ be real variables $s_{k} \in \mathbb{R}^{D}$ assigned to the chosen basis of the homology $H^{1}(\Gamma, \mathbb{Z})$. Also let $p_{i}$, for $i=1, \ldots, n$ be real variables $p_{i} \in \mathbb{R}^{D}$ associated to the edges of $\Gamma$. Let $q_{i}(p)$ denote the quadratic form

$$
q_{i}(p)=p_{i}^{2}-m_{i}^{2},
$$

for fixed parameters $m_{i}>0$. These correspond to the Feynman propagators

$$
\frac{1}{q_{i}}=\frac{1}{p_{i}^{2}-m_{i}^{2}}
$$

for a scalar field theory, associated by the Feynman rules to the edges of the graph. One can make a change of variables

$$
p_{i}=u_{i}+\sum_{k=1}^{\ell} \eta_{i k} s_{k}, \quad \text { with the constraint } \sum_{i=0}^{n} t_{i} u_{i} \eta_{i k}=0 .
$$

Then we have the following result.

Lemma 3.2. The following identity holds

$$
\int \frac{1}{\left(\sum_{i=0}^{n} t_{i} q_{i}\right)^{n}} d^{D} s_{1} \cdots d^{D} s_{\ell}=C_{\ell, n} \operatorname{det}\left(M_{\Gamma}(t)\right)^{-D / 2}\left(\sum_{i=0}^{n} t_{i}\left(u_{i}^{2}-m_{i}^{2}\right)\right)^{-n+D \ell / 2}
$$

Proof. After the change of variables, the left hand side reads

$$
\int \frac{d^{D} s_{1} \cdots d^{D} s_{\ell}}{\left(\sum_{i=0}^{n} t_{i}\left(u_{i}^{2}-m_{i}^{2}\right)+\sum_{k r}\left(M_{\Gamma}\right)_{k r} s_{k} s_{r}\right)^{n}} .
$$

The integral can then be reduced by a change of variables that diagonalizes the matrix $M_{\Gamma}$ to an integral of the form

$$
\int \frac{d^{D} x_{1} \cdots d^{D} x_{\ell}}{\left(a-\sum_{k} \lambda_{k} x_{k}^{2}\right)^{n}}=C_{\ell, n} a^{-n+D \ell / 2} \prod_{k=1}^{\ell} \lambda_{k}^{-D / 2},
$$

with

$$
C_{\ell, n}=\int \frac{d^{D} x_{1} \cdots d^{D} x_{\ell}}{\left(1-\sum_{k} x_{k}^{2}\right)^{n}}
$$

This is the basis for the well known formula that relates the computation of Feynman integrals to periods, used in [8. In fact, we have the following.

Corollary 3.3. In the case of graphs where the number of edges and the number of loops are related by $n=D \ell / 2$, the Feynman integral is computed by

$$
\int \frac{d^{D} s_{1} \cdots d^{D} s_{\ell}}{q_{0} \cdots q_{n}}=C_{\ell, n} \int_{\Sigma_{n}} \frac{d t_{0} \cdots d t_{n-1}}{\Psi_{\Gamma}\left(t_{0}, \ldots, t_{n}\right)^{D / 2}},
$$


where $t_{n}=1-\sum_{i=0}^{n-1} t_{i}$ and

$$
\Psi_{\Gamma}(t)=\operatorname{det}\left(M_{\Gamma}(t)\right) .
$$

Proof. Notice that, in the case of graphs with $n=D \ell / 2$, the integration (3.9) reduces to

$$
\int \frac{d^{D} s_{1} \cdots d^{D} s_{\ell}}{\left(\sum_{i=0}^{n} t_{i} q_{i}\right)^{n}}=C_{\ell, n} \operatorname{det}\left(M_{\Gamma}(t)\right)^{-D / 2}
$$

We now consider a modified version of this construction, where we deal with graphs that have both bosonic and fermionic legs, and we maintain the distinction between these two types at all stages by assigning to them different sets of ordinary and Grassmann variables. Strictly from the physicists point of view this is an unnecessary complication, because the formulae we recalled in this section adapt to compute Feynman integrals also in theories with fermionic fields, but from the mathematical viewpoint this procedure will provide us with a natural way of constructing an interesting class of supermanifolds with associated periods.

3.2. The case of Grassmann variables. Consider now the case of Feynman propagators and Feynman diagrams that come from theories with both bosonic and fermionic fields. This means that, in addition to terms of the form (1.1), the Lagrangian also contains fermion interaction terms. The form of such terms is severely constrained (see e.g. [20, $\S 5.3$ ): for instance, in dimension $D=4$ renormalizable interaction terms can only involve at most two fermion and one boson field.

The perturbative expansion for such theories corresponsingly involve graphs $\Gamma$ with two different types of edges: fermionic and bosonic edges. The Feynman rules assign to each bosonic edge a propagator of the form (3.8) and to fermionic edges a propagator

$$
i \frac{\mathrm{p}+m}{p^{2}-m^{2}}=\frac{i}{\mathrm{p}-m} \text {. }
$$

Notice that in physically significant theories one would have $i(\not p-m)^{-1}$ with $\not p=p^{\mu} \gamma_{\mu}$, but for simplicity we work here with propagators of the form (3.13), without tensor indices.

In the following we use the notation

$$
q(p)=p^{2}-m^{2}, \quad \mathrm{q}(p)=i(\mathrm{p}+m)
$$

for the quadratic and linear forms that appear in the propagators (3.8) and (3.13). In the following, again just to simplify notation, we also drop the mass terms in the propagator (i.e. we set $m=0$ ) and ignore the resulting infrared divergence problem. The reader can easily reinstate the masses whenever needed.

Thus, the terms of the form $\left(q_{1} \cdots q_{n}\right)^{-1}$, which we encountered in the purely bosonic case, are now replaced by terms of the form

$$
\frac{\mathrm{q}_{1} \cdots \mathrm{q}_{f}}{q_{1} \cdots q_{n}}
$$

where $n=\# E(\Gamma)$ is the total number of edges in the graph and $f=\# E_{f}(\Gamma)$ is the number of fermionic edges.

We first prove an analog of Lemma 3.1 where we now introduce an extra set of Grassmann variables associated to the fermionic edges. The derivation we present suffers from a kind of "fermion doubling problem", in as each fermionic edge contributes an ordinary integration variables, which essentially account for the denominator $q_{i}$ in (3.13) and (3.15), as well as a pair of Grassman variables accounting for the numerator $q_{i}$ in (3.13) and (3.15). 
Let $\mathcal{Q}_{f}$ denote the $2 f \times 2 f$ antisymmetric matrix

$$
\mathcal{Q}_{f}=\left(\begin{array}{ccccccc}
0 & \mathrm{q}_{1} & 0 & 0 & \cdots & 0 & 0 \\
-\mathrm{q}_{1} & 0 & 0 & 0 & \cdots & 0 & 0 \\
0 & 0 & 0 & \mathrm{q}_{2} & \cdots & 0 & 0 \\
0 & 0 & -\mathrm{q}_{2} & 0 & \cdots & 0 & 0 \\
\vdots & & & & \ldots & & \vdots \\
0 & 0 & 0 & 0 & \cdots & 0 & \mathrm{q}_{f} \\
0 & 0 & 0 & 0 & \cdots & -\mathrm{q}_{f} & 0
\end{array}\right) .
$$

Lemma 3.4. Let $\Sigma_{n \mid 2 f}$ denote the superspace $\Sigma_{n} \times \mathbb{A}^{0 \mid 2 f}$. Then the following identity holds:

$$
\frac{\mathrm{q}_{1} \cdots \mathrm{q}_{f}}{q_{1} \cdots q_{n}}=K_{n, f} \int_{\Sigma_{n \mid 2 f}} \frac{d t_{1} \cdots d t_{n-1} d \theta_{1} \cdots d \theta_{2 f}}{\left(t_{1} q_{1}+\cdots t_{n} q_{n}+\frac{1}{2} \theta^{t} \mathcal{Q}_{f} \theta\right)^{n-f}}
$$

with

$$
K_{n, f}=\frac{2^{f}(n-1) !}{\prod_{k=1}^{f}(-n+f-k+1)} .
$$

Proof. We first show that the following identity holds for integration in the Grassmann variables $\theta=\left(\theta_{1}, \ldots, \theta_{2 f}\right)$ :

$$
\int \frac{d \theta_{1} \cdots d \theta_{2 f}}{\left(1+\frac{1}{2} \theta^{t} \mathcal{Q}_{f} \theta\right)^{\alpha}}=\frac{f !}{2^{f}}\left(\begin{array}{c}
-\alpha \\
f
\end{array}\right) \mathrm{q}_{1} \cdots \mathrm{q}_{f} .
$$

In fact, we expand using the Taylor series

$$
(1+x)^{\beta}=\sum_{k=0}^{\infty}\left(\begin{array}{l}
\beta \\
k
\end{array}\right) x^{k}
$$

and the identity

$$
\frac{1}{2} \theta^{t} \mathcal{Q}_{f} \theta=\sum_{i=1}^{f} \mathrm{q}_{i} \theta_{2 i-1} \theta_{2 i}
$$

together with the fact that the degree zero variables $x_{i}=\theta_{2 i-1} \theta_{2 i}$ commute, to obtain

$$
\left(1+\frac{1}{2} \theta^{t} \mathcal{Q}_{f} \theta\right)^{-\alpha}=\sum_{k=0}^{\infty}\left(\begin{array}{c}
-\alpha \\
k
\end{array}\right)\left(\sum_{i=1}^{f} \mathrm{q}_{i} \theta_{2 i-1} \theta_{2 i}\right)^{k} .
$$

The rules of Grassmann integration then imply that only the coefficient of $\theta_{1} \cdots \theta_{2 f}$ remains as a result of the integration. This gives (3.18).

For simplicity of notation, we then write $T=t_{1} q_{1}+\cdots t_{n} q_{n}$, so that we have

$$
\begin{gathered}
\int_{\Sigma_{n \mid 2 f}} \frac{1}{\left(t_{1} q_{1}+\cdots t_{n} q_{n}+\frac{1}{2} \theta^{t} \mathcal{Q}_{f} \theta\right)^{n-f}} d t_{1} \cdots d t_{n-1} d \theta_{1} \cdots d \theta_{2 f}= \\
\frac{f !}{2^{f}}\left(\begin{array}{c}
-n+f \\
f
\end{array}\right) \mathrm{q}_{1} \cdots \mathrm{q}_{f} \int_{\Sigma_{n}} T^{-n+f} T^{-f} d t_{1} \cdots d t_{n-1} \\
=\frac{f !}{2^{f}}\left(\begin{array}{c}
-n+f \\
f
\end{array}\right) \mathrm{q}_{1} \cdots \mathrm{q}_{f} \int_{\Sigma_{n}} \frac{d t_{1} \cdots d t_{n-1}}{\left(t_{1} q_{1}+\cdots+t_{n} q_{n}\right)^{n}}=\frac{f !}{2^{f}(n-1) !}\left(\begin{array}{c}
-n+f \\
f
\end{array}\right) \frac{\mathrm{q}_{1} \cdots \mathrm{q}_{f}}{q_{1} \cdots q_{n}} .
\end{gathered}
$$


3.3. Graphs with fermionic legs. Consider now the case of graphs that have both bosonic and fermionic legs. We mimic the procedure described above, but by using both ordinary and Grassmann variables in the process.

We divide the edge indices $i=1, \ldots, n$ into two sets $i_{b}=1, \ldots, n_{b}$ and $i_{f}=1, \ldots, n_{f}$, with $n=n_{b}+n_{f}$, respectively labeling the bosonic and fermionic legs. Consequently, given a choice of a basis for the first homology of the graph, indexed as above by $r=1, \ldots, \ell$, we replace the matrix $\eta_{i r}$ of (3.5), with a matrix of the form

$$
\left(\begin{array}{ll}
\eta_{i_{f} r_{f}} & \eta_{i_{f} r_{b}} \\
\eta_{i_{b} r_{f}} & \eta_{i_{b} r_{b}}
\end{array}\right)
$$

Here the loop indices $r=1, \ldots, \ell$ are at first divided into three sets $\left\{1, \ldots, \ell_{f f}\right\}$, labelling the loops consisiting of only fermionic edges, $\left\{1, \ldots, \ell_{b b}\right\}$ labelling the loops consisting of only bosonic edges, and the remaning variables $\left\{1, \ldots, \ell_{b f}=\ell-\left(\ell_{f f}+\ell_{b b}\right)\right\}$ for the loops that contain both fermionic and bosonic edges. We then introduce two sets of momentum variables: ordinary variables $\mathrm{s}_{r_{b}} \in \mathbb{A}^{D \mid 0}$, with $r_{b}=1, \ldots, \ell_{b}=\ell_{b b}+\ell_{b f}$, and Grassmann variables $\sigma_{r_{f}} \in \mathbb{A}^{0 \mid D}$ with $r_{f}=1, \ldots, \ell_{f}=\ell_{f f}+\ell_{b f}$. That is, we assign to each purely fermionic loop a Grassmann momentum variable, to each purely bosonic loop an ordinary momentum variable, and to the loops containing both fermionic and bosonic legs a pair $\left(\mathrm{s}_{r}, \sigma_{r}\right)$ of an ordinary and a Grassman variable. In (3.19) above we write $r_{f}$ and $r_{b}$, respectively, for the indexing sets of these Grassmann and ordinary variables.

We then consider a change of variables

$$
\mathrm{p}_{i_{b}}=\mathrm{u}_{i_{b}}+\sum_{r_{f}} \eta_{i_{b} r_{f}} \sigma_{r_{f}}+\sum_{r_{b}} \eta_{i_{b} r_{b}} \mathrm{~s}_{r_{b}}, \quad \mathrm{p}_{i_{f}}=\mathrm{u}_{i_{f}}+\sum_{r_{f}} \eta_{i_{f} r_{f}} \sigma_{r_{f}}+\sum_{r_{b}} \eta_{i_{f} r_{b}} \mathrm{~s}_{r_{b}} .
$$

analogous to the one used before, where now, for reasons of homogeneity, we need to assume that the $\eta_{i r_{f}}$ are of degree one and the $\eta_{i r_{b}}$ are of degree zero, since the $\mathrm{p}_{i}$ are even (ordinary) variables.

We apply the change of variables (3.20) to the expression

$$
\sum_{i} t_{i} p_{i}^{2}+\sum_{i_{f}} \theta_{2 i_{f}-1} \theta_{2 i_{f}} \mathrm{p}_{i_{f}}
$$

We assume again, as in the purely bosonic case ( $c f .(18.35)$ of [6]), the relations

$$
\sum_{i} t_{i} \mathrm{u}_{i} \eta_{i r}=0
$$

for each loop variable $r=r_{b}$ and $r=r_{f}$.

We can then rewrite (3.21) in the form

$$
\begin{gathered}
\sum_{i} t_{i} u_{i}^{2}+\sum_{i_{f}} \theta_{2 i_{f}-1} \theta_{2 i_{f}} \mathrm{u}_{i_{f}} \\
+\sum_{r_{b}, r_{b}^{\prime}}\left(\sum_{i} t_{i} \eta_{i r_{b}} \eta_{i r_{b}^{\prime}}\right) \mathrm{s}_{r_{b}} \mathrm{~s}_{r_{b}^{\prime}}-\sum_{r_{f} r_{f}^{\prime}}\left(\sum_{i} t_{i} \eta_{i r_{f}} \eta_{i r_{f}^{\prime}}\right) \sigma_{r_{f}} \sigma_{r_{f}^{\prime}} \\
+\sum_{r_{b} r_{f}}\left(\left(\sum_{i} t_{i} \eta_{i r_{b}} \eta_{i r_{f}}\right) \mathrm{s}_{r_{b}} \sigma_{r_{f}}-\sigma_{r_{f}}^{\tau} \mathrm{s}_{r_{b}}^{\tau}\left(\sum_{i} t_{i} \eta_{i r_{f}} \eta_{i r_{b}}\right)\right) \\
+\sum_{r_{b}}\left(\sum_{i_{f}} \theta_{2 i_{f}-1} \theta_{2 i_{f}} \eta_{i_{f} r_{b}}\right) \mathrm{s}_{r_{b}}+\sum_{r_{f}}\left(\sum_{i_{f}} \theta_{2 i_{f}-1} \theta_{2 i_{f}} \eta_{i_{f} r_{f}}\right) \sigma_{r_{f}} .
\end{gathered}
$$

Notice the minus sign in front of the quadratic term in the $\sigma_{r_{f}}$, since for order-one variables $\sigma_{r_{f}} \eta_{i r_{f}^{\prime}}=-\eta_{i r_{f}^{\prime}} \sigma_{r_{f}}$. We write the above in the simpler notation

$$
T+\mathrm{s}^{\tau} M_{b}(t) \mathrm{s}-\sigma^{\tau} M_{f}(t) \sigma+\sigma^{\tau} M_{f b}(t) \mathrm{s}-\mathrm{s}^{\tau} M_{b f}(t) \sigma+N_{b}(\theta) \mathrm{s}+N_{f}(\theta) \sigma,
$$


where $\tau$ denotes transposition, $\mathrm{s}=\left(\mathrm{s}_{r_{b}}\right), \sigma=\left(\sigma_{r_{f}}\right)$, and

$$
\begin{aligned}
& T=\sum_{i} t_{i} u_{i}^{2}+\sum_{i_{f}} \theta_{2 i_{f}-1} \theta_{2 i_{f}} u_{i_{f}}, \\
& M_{b}(t)=\sum_{i} t_{i} \eta_{i r_{b}} \eta_{i r_{b}^{\prime}}, \\
& M_{f}(t)=\sum_{i} t_{i} \eta_{i r_{f}} \eta_{i r_{f}^{\prime}}=-M_{f}(t)^{\tau}, \\
& M_{f b}(t)=\sum_{i} t_{i} \eta_{i r_{b}} \eta_{i r_{f}}, \\
& N_{b}(\theta)=\sum_{i_{f}} \theta_{2 i_{f}-1} \theta_{2 i_{f}} \eta_{i_{f}} r_{b}, \\
& N_{f}(\theta)=\sum_{i_{f}} \theta_{2 i_{f}-1} \theta_{2 i_{f}} \eta_{i_{f} r_{f}} .
\end{aligned}
$$

Since the $\eta_{i, r_{f}}$ are of degree one and the $\eta_{i, r_{b}}$ of degree zero, the matrices $M_{b}$ and $M_{f}$ are of degree zero, the $M_{b f}$ and $M_{f b}$ of degree one, while the $N_{b}$ and $N_{f}$ are, respectively, of degree zero and one. Thus, the expression (3.22) is of degree zero. Notice that, since the $\eta_{i r_{f}}$ are of order one, the matrix $M_{f}(t)$ is antisymmetric. We also set $M_{b f}(t)=M_{f b}(t)=$ $M_{f b}(t)^{\tau}$.

We then consider an integral of the form

$$
\int \frac{d^{D} \mathrm{~s}_{1} \cdots d^{D} \mathrm{~s}_{\ell_{b}} d^{D} \sigma_{1} \cdots d^{D} \sigma_{\ell_{f}}}{\left(\sum_{i} t_{i} p_{i}^{2}+\sum_{i_{f}} \theta_{2 i_{f}-1} \theta_{2 i_{f}} \mathrm{p}_{i_{f}}\right)^{n-f}}=
$$

$$
\int \frac{d^{D} \mathrm{~s}_{1} \cdots d^{D} \mathrm{~s}_{\ell_{b}} d^{D} \sigma_{1} \cdots d^{D} \sigma_{\ell_{f}}}{\left(T+\mathrm{s}^{\tau} M_{b}(t) \mathrm{s}+N_{b}(\theta) \mathrm{s}-\sigma^{\tau} M_{f}(t) \sigma+\sigma^{\tau} M_{f b}(t) \mathrm{s}-\mathrm{s}^{\tau} M_{f b}(t)^{\tau} \sigma+N_{f}(\theta) \sigma\right)^{n-f}},
$$

where the $d^{D} \sigma_{i}=d \sigma_{i 1} \cdots d \sigma_{i D}$ are Grassmann variables integrations and the $d^{D} \mathrm{~s}_{i}$ are ordinary integrations.

Recall that for Grassmann variables we have the following change of variable formula.

Lemma 3.5. Suppose given an invertible antisymmetric $N \times N$-matrix $A$ with entries of degree zero and an $N$-vector $J$ with entries of degree one. Then we have

$$
\sigma^{\tau} A \sigma+\frac{1}{2}\left(J^{\tau} \sigma-\sigma^{\tau} J\right)=\eta^{\tau} A \eta+\frac{1}{4} J^{\tau} A^{-1} J,
$$

for $\eta=\sigma-\frac{1}{2} A^{-1} J$.

Proof. The result is immediate: since $A^{\tau}=-A$, we simply have

$$
\eta^{\tau} A \eta=\sigma^{\tau} A \sigma+\frac{1}{2} J^{\tau} \sigma-\frac{1}{2} \sigma^{\tau} J-\frac{1}{4} J^{\tau} A^{-1} J .
$$

We then use this change of variable to write

$$
\begin{gathered}
-\sigma^{\tau} M_{f}(t) \sigma+\sigma^{\tau} M_{f b}(t) \mathrm{s}-\mathrm{s}^{\tau} M_{f b}(t)^{\tau} \sigma+\frac{1}{2}\left(\sigma^{\tau} N_{f}(\theta)-N_{f}(\theta)^{\tau} \sigma\right)= \\
-\eta^{\tau} M_{f}(t) \eta-\frac{1}{4}\left(M_{f b}(t) \mathrm{s}+\frac{1}{2} N_{f}(\theta)\right)^{\tau} M_{f}(t)^{-1}\left(M_{f b}(t) \mathrm{s}+\frac{1}{2} N_{f}(\theta)\right)
\end{gathered}
$$

with

$$
\eta=\sigma-\frac{1}{2} M_{f}(t)^{-1}\left(M_{f b}(t) \mathrm{s}+\frac{1}{2} N_{f}(\theta)\right) .
$$

We have

$$
\begin{gathered}
\frac{1}{4}\left(M_{f b}(t) \mathrm{s}+\frac{1}{2} N_{f}(\theta)\right)^{\tau} M_{f}(t)^{-1}\left(M_{f b}(t) \mathrm{s}+\frac{1}{2} N_{f}(\theta)\right)= \\
\frac{1}{4} \mathrm{~s}^{\tau} M_{b f}(t) M_{f}(t)^{-1} M_{f b}(t) \mathrm{s}+\frac{1}{8}\left(N_{f}(\theta)^{\tau} M_{f}(t)^{-1} M_{f b}(t) \mathrm{s}+\mathrm{s}^{\tau} M_{b f}(t) M_{f}(t)^{-1} N_{f}(\theta)\right)
\end{gathered}
$$


We then let

$$
+\frac{1}{16} N_{f}(\theta)^{\tau} M_{f}(t)^{-1} N_{f}(\theta) .
$$

$$
U(t, \theta, \mathrm{s}):=T+C(t, \theta)+\mathrm{s}^{\tau} A_{b}(t) \mathrm{s}+B_{b}(t, \theta) \mathrm{s},
$$

where

$$
\begin{aligned}
& A_{b}(t)=M_{b}(t)-\frac{1}{4} M_{b f}(t) M_{f}(t)^{-1} M_{f b}(t) \\
& B_{b}(t, \theta)=N_{b}(\theta)-\frac{1}{4} N_{f}(\theta)^{\tau} M_{f}(t)^{-1} M_{f b}(t) \\
& C(t, \theta)=-\frac{1}{16} N_{f}(\theta)^{\tau} M_{f}(t)^{-1} N_{f}(\theta) .
\end{aligned}
$$

Thus, we write the denominator of (3.24) in the form

$$
U(t, \theta, \mathrm{s})^{n-f}\left(1+\frac{1}{2} \eta^{\tau} X_{f}(t, \theta, \mathrm{s}) \eta\right)^{n-f}
$$

where we use the notation

$$
X_{f}(t, \theta, \mathrm{s}):=2 U(t, \theta, \mathrm{s})^{-1} M_{f}(t) .
$$

Thus, the Grassmann integration in (3.24) gives, as in Lemma 3.4.

$$
\int \frac{d^{D} \eta_{1} \cdots d^{D} \eta_{\ell_{f}}}{\left(1+\frac{1}{2} \eta^{\tau} X_{f}(t, \theta, \mathrm{s}) \eta\right)^{n-f}}=C_{n, f, \ell_{f}} \frac{2^{D \ell_{f} / 2}}{U(t, \theta, \mathrm{s})^{D \ell_{f} / 2}} \operatorname{det}\left(M_{f}(t)\right)^{D / 2},
$$

where $C_{n, f, \ell_{f}}$ is a combinatorial factor obtained as in Lemma 3.4

We then proceed to the remaining ordinary integration in (3.24). We have, dropping a multiplicative constant,

$$
\operatorname{det}\left(M_{f}(t)\right)^{D / 2} \int \frac{d^{D} \mathrm{~s}_{1} \cdots d^{D} \mathrm{~s}_{\ell_{b}}}{U(t, \theta, \mathrm{s})^{n-f+D \ell_{f} / 2}} .
$$

This now can be computed as in the original case we reviewed in 33.1 above. We use the change of variables $\mathrm{v}=\mathrm{s}+\frac{1}{2} M_{b}(t)^{-1} N_{b}(\theta)^{\tau}$. We then have

$$
\mathrm{v}^{\tau} A_{b}(t) \mathrm{v}=\mathrm{s}^{\tau} A_{b}(t) \mathrm{s}+\frac{1}{2} \mathrm{~s}^{\tau} B_{b}(t, \theta)^{\tau}+\frac{1}{2} B_{b}(t, \theta) \mathrm{s}+\frac{1}{4} B_{b}(t, \theta) A_{b}(t)^{-1} B_{b}(t, \theta)^{\tau},
$$

where $A_{b}(t)^{\tau}=A_{b}(t)$ and $\left(B_{b}(t, \theta) \mathrm{s}\right)^{\tau}=B_{b}(t, \theta) \mathrm{s}$.

We then rewrite (3.33) in the form

$$
\operatorname{det}\left(M_{f}(t)\right)^{D / 2} \int \frac{d^{D} \mathrm{v}_{1} \cdots d^{D} \mathrm{v}_{\ell_{b}}}{\left(T+C-\frac{1}{4} B_{b} A_{b}^{-1} B_{b}^{\tau}+\mathrm{v}^{\tau} A_{b} \mathrm{v}\right)^{n-f+D \ell_{f} / 2}} .
$$

Set then

$$
\tilde{T}(t, \theta)=T(t, \theta)+C(t, \theta)-\frac{1}{4} B_{b}(t, \theta) A_{b}^{-1}(t) B_{b}(t, \theta)^{\tau},
$$

so that we write the above as

$$
\frac{\operatorname{det}\left(M_{f}(t)\right)^{D / 2}}{\tilde{T}(t, \theta)^{n-f+D \ell_{f} / 2}} \int \frac{d^{D} \mathrm{v}_{1} \cdots d^{D} \mathrm{v}_{\ell_{b}}}{\left(1+\mathrm{v}^{\tau} X_{b}(t, \theta) \mathrm{v}\right)^{n-f+D \ell_{f} / 2}},
$$

with

$$
X_{b}(t, \theta)=\tilde{T}(t, \theta)^{-1} A_{b}(t) .
$$

Then, up to a multiplicative constant, the integral gives

$$
\tilde{T}^{-n+f-\frac{D \ell_{f}}{2}+\frac{D \ell_{b}}{2}} \frac{\operatorname{det}\left(M_{f}(t)\right)^{D / 2}}{\operatorname{det}\left(A_{b}(t)\right)^{D / 2}} .
$$


Consider first the term

$$
\frac{\operatorname{det}\left(M_{f}(t)\right)^{D / 2}}{\operatorname{det}\left(A_{b}(t)\right)^{D / 2}}
$$

in (3.37) above. This can be identified with a Berezinian. In fact, we have

$$
\frac{\operatorname{det}\left(M_{f}(t)\right)^{D / 2}}{\operatorname{det}\left(M_{b}(t)-\frac{1}{4} M_{f b}(t) M_{f}(t)^{-1} M_{f b}(t)\right)^{D / 2}}=\operatorname{Ber}(\mathcal{M}(t))^{-D / 2},
$$

where

$$
\mathcal{M}(t)=\left(\begin{array}{cc}
M_{b}(t) & \frac{1}{2} M_{f b}(t) \\
\frac{1}{2} M_{b f}(t) & M_{f}(t)
\end{array}\right)
$$

We now look more closely at the remaining term $\tilde{T}^{-n+f-\frac{D \ell_{f}}{2}+\frac{D \ell_{b}}{2}}$ in (3.37). We know from (3.36), (3.29), and (3.23) that we can write $\tilde{T}(t, \theta)$ in the form

$$
\tilde{T}(t, \theta)=\sum_{i} u_{i}^{2} t_{i}+\sum_{j} \mathrm{u}_{i} \theta_{2 j-1} \theta_{2 j}+\sum_{i<j} C_{i j}(t) \theta_{2 i-1} \theta_{2 i} \theta_{2 j-1} \theta_{2 j},
$$

where the first sum is over all edges and the other two sums are over fermionic edges. We set $\lambda_{i}=\theta_{2 i-1} \theta_{2 i}$. Using a change of variables $\tilde{\lambda}_{i}=\lambda_{i}+\frac{1}{2} C \mathrm{u}$, we rewrite the above as

$$
\tilde{T}(t, \theta)=\sum_{i} u_{i}^{2} t_{i}-\frac{1}{4} \mathrm{u}^{\tau} C \mathrm{u}+\sum_{i<j} C_{i j} \eta_{2 i-1} \eta_{2 i} \eta_{2 j-1} \eta_{2 j}
$$

with $\tilde{\lambda}_{i}=\eta_{2 i-1} \eta_{2 i}$. We denote by

$$
\hat{T}(t)=\sum_{i} u_{i}^{2} t_{i}-\frac{1}{4} \mathrm{u}^{\tau} C \mathrm{u}
$$

and we write

$$
\tilde{T}^{-\alpha}=\hat{T}^{-\alpha} \sum_{k=0}^{\infty}\left(\begin{array}{c}
-\alpha \\
k
\end{array}\right)\left(\frac{\frac{1}{2} \tilde{\lambda}^{\tau} C \tilde{\lambda}}{\hat{T}}\right)^{k}
$$

where we use the notation $\frac{1}{2} \tilde{\lambda}^{\tau} C \tilde{\lambda}=\sum_{i<j} C_{i j} \eta_{2 i-1} \eta_{2 i} \eta_{2 j-1} \eta_{2 j}$.

Thus, we can write the Feynman integral in the form

$$
\begin{gathered}
\int \frac{\mathrm{q}_{1} \cdots \mathrm{q}_{f}}{q_{1} \cdots q_{n}} d^{D} s_{1} \cdots d^{D} s_{\ell_{b}} d^{D} \sigma_{1} \cdots d^{D} \sigma_{\ell_{f}}= \\
\kappa \int_{\Sigma_{n \mid 2 f}} \frac{\Lambda(t) \eta_{1} \cdots \eta_{2 f}}{\hat{T}(t)^{n-\frac{f}{2}+\frac{D}{2}\left(\ell_{f}-\ell_{b}\right)} \operatorname{Ber}(\mathcal{M}(t))^{D / 2}} d t_{1} \cdots d t_{n} d \eta_{1} \cdots d \eta_{2 f},
\end{gathered}
$$$$
\text { where } \Lambda(t) \text { is } \hat{T}^{f / 2} \text { times the coefficient of } \eta_{1} \cdots \eta_{2 f} \text { in the expansion }
$$$$
\sum_{k=0}^{\infty}\left(\begin{array}{c}
-\alpha \\
k
\end{array}\right)\left(\frac{\frac{1}{2} \tilde{\lambda}^{\tau} C \tilde{\lambda}}{\hat{T}}\right)^{k}
$$

More explicitly, this term is of the form

$$
\Lambda(t)=\sum C_{i_{1} i_{2}}(t) \cdots C_{i_{f-1} i_{f}}(t),
$$

over indices $i_{a}$ with $i_{2 a-1}<i_{2 a}$ and for $k=f / 2$. The multiplicative constant in front of the integral on the right hand side above is given by

$$
\kappa=\left(\begin{array}{c}
-n+f-\frac{D}{2}\left(\ell_{f}-\ell_{b}\right) \\
f / 2
\end{array}\right) .
$$

We then obtain the following result. 
Theorem 3.6. Suppose given a graph $\Gamma$ with $n$ edges, of which $f$ fermionic and $b=n-f$ bosonic. Assume that there exists a choice of a basis for $H_{1}(\Gamma)$ satisfying the condition

$$
n-\frac{f}{2}+\frac{D}{2}\left(\ell_{f}-\ell_{b}\right)=0 .
$$

Then the following identity holds:

$$
\int \frac{\mathrm{q}_{1} \cdots \mathrm{q}_{f}}{q_{1} \cdots q_{n}} d^{D} s_{1} \cdots d^{D} s_{\ell_{b}} d^{D} \sigma_{1} \cdots d^{D} \sigma_{\ell_{f}}=\int_{\Sigma_{n}} \frac{\Lambda(t)}{\operatorname{Ber}(\mathcal{M}(t))^{D / 2}} d t_{1} \cdots d t_{n} .
$$

Proof. This follows directly from (3.41), after imposing $n-\frac{f}{2}+\frac{D}{2}\left(\ell_{f}-\ell_{b}\right)=0$ and performing the Grassmann integration of the resulting term

$$
\int_{\Sigma_{n \mid 2 f}} \frac{\Lambda(t) \eta_{1} \cdots \eta_{2 f}}{\operatorname{Ber}(\mathcal{M}(t))^{D / 2}} d t_{1} \cdots d t_{n} d \eta_{1} \cdots d \eta_{2 f}
$$

3.4. Graph supermanifolds. The result of the previous section shows that we have an analog of the period integral

given by the similar expression

$$
\int_{\Sigma_{n}} \frac{d t_{1} \cdots d t_{n}}{\operatorname{det}\left(M_{\Gamma}(t)\right)^{D / 2}}
$$

$$
\int_{\Sigma_{n}} \frac{\Lambda(t)}{\operatorname{Ber}(\mathcal{M}(t))^{D / 2}} d t_{1} \cdots d t_{n}
$$

Again we see that, in this case, divergences arise from the intersections between the domain of integration given by the simplex $\Sigma_{n}$ and the subvariety of $\mathbb{P}^{n-1}$ defined by the solutions of the equation

$$
\frac{\operatorname{Ber}(\mathcal{M}(t))^{D / 2}}{\Lambda(t)}=0
$$

Lemma 3.7. For generic graphs, the set of zeros of (3.46) defines a hypersurface in $\mathbb{P}^{n}$, hence a divisor in $\mathbb{P}^{n-1 \mid 2 f}$ of dimension $(n-2 \mid 2 f)$. The support of this divisor is the same as that of the principal divisor defined by $\operatorname{Ber}(\mathcal{M}(t))$.

Proof. The generic condition on graphs is imposed to avoid the cases with $M_{f}(t) \equiv 0$. Thus, suppose given a pair $(\Gamma, B)$ that is generic, in the sense that $M_{f}(t)$ is not identically zero. The equation (3.46) is satisfied by solutions of

$$
\operatorname{det}\left(M_{b}(t)-\frac{1}{4} M_{b f}(t) M_{f}(t)^{-1} M_{f b}(t)\right)=0
$$

and by poles of $\Lambda(t)$. Using the formulae (3.29) and (3.23) we see that the denominator of $\Lambda(t)$ is given by powers of $\operatorname{det}\left(M_{f}(t)\right)$ and $\operatorname{det}\left(A_{b}(t)\right)=\operatorname{det}\left(M_{b}(t)-\frac{1}{4} M_{b f}(t) M_{f}(t)^{-1} M_{f b}(t)\right)$. Thus, the set of solutions of (3.46) is the union of zeros and poles of $\operatorname{Ber}(\mathcal{M}(t))$. The multiplicities are given by the powers of these determinants that appear in $\Lambda(t) \operatorname{Ber}(\mathcal{M}(t))^{-D / 2}$.

Definition 3.8. Let $\Gamma$ be a graph with bosonic and fermionic edges and $B$ a choice of a basis of $H_{1}(\Gamma)$. We denote by $\mathcal{X}_{(\Gamma, B)} \subset \mathbb{P}^{n-1 \mid 2 f}$ the locus of zeros and poles of $\operatorname{Ber}(\mathcal{M}(t))=$ 0 . We refer to $\mathcal{X}_{(\Gamma, B)}$ as the graph supermanifold.

In the degenerate cases of graphs such that $M_{f}(t) \equiv 0$, we simply set $\mathcal{X}_{(\Gamma, B)}=\mathbb{P}^{n-1 \mid 2 f}$. Examples of this sort are provided by data $(\Gamma, B)$ such that there is only one loop in $B$ containing fermionic edges. Other special cases arise when we consider graphs with only bosonic or only fermionic edges. In the first case, we go back to the original calculation without Grassmann variables and we therefore simply recover $\mathcal{X}_{(\Gamma, B)}=$ 

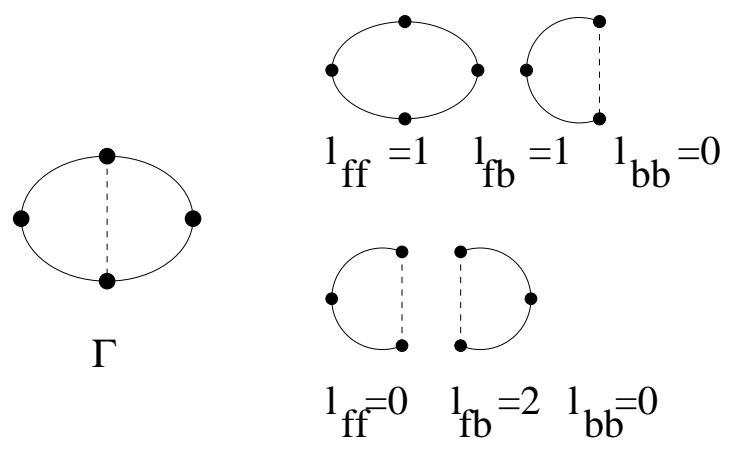

Figure 1. Choices of a basis for $H_{1}(\Gamma)$.



FIgURE 2. A graph with a basis of $H_{1}(\Gamma)$ satisfying (3.42).

$X_{\Gamma}=\left\{t: \operatorname{det}\left(M_{b}(t)\right)=0\right\} \subset \mathbb{P}^{n-1 \mid 0}$. In the case with only fermionic edges, we have $\operatorname{det}\left(M_{b}(t)-\frac{1}{4} M_{b f}(t) M_{f}(t)^{-1} M_{f b}(t)\right) \equiv 0$ since both $M_{b}(t)$ and $M_{b f}(t)$ are identically zero. It is then natural to simply assume that, in such cases, the graph supermanifold is simply given by $\mathcal{X}_{(\Gamma, B)}=\mathbb{P}^{f-1 \mid 2 f}$.

3.5. Examples from Feynman graphs. We still need to check that the condition (3.42) we imposed on the graph is satisfied by some classes of interesting graphs. First of all, notice that the condition does not depend on the graph alone, but on the choice of a basis for $H_{1}(\Gamma)$. The same graph can admit choices for which (3.42) is satisfied and others for which it fails to hold. For example, consider the graph illustrated in Figure1, for a theory in dimension $D=6$, where we denoted bosonic edges by the dotted line and fermionic ones by the full line. There exists a choice of a basis of $H_{1}(\Gamma)$ for which (3.42) is satisfied, as the first choice in the figure shows, while not all choices satisfy this condition, as one can see in the second case.

One can see easily that one can construct many examples of graphs that admit a basis of $H_{1}(\Gamma)$ satisfying (3.42). For instance, the graph in Figure 2 is a slightly more complicated example in $D=6$ of a graph satisfying the condition. Again we used dotted lines for the bosonic edges and full lines for the fermionic ones.

Let us consider again the example of the very simple graph of Figure 1, with the first choice of the basis $B$ for $H_{1}(\Gamma)$. This has two generators, one of them a loop made of fermionic edges and the second a loop containing both fermionic and bosonic edges. Let 


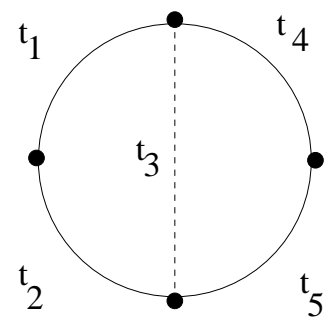

Figure 3. Edge variables.

us assign the ordinary variables $t_{i}$ with $i=1, \ldots, 5$ to the edges as in Figure 3 We then have

$$
M_{b}(t)=t_{1}+t_{2}+t_{3}
$$

since only the second loop in the basis contains bosonic edges, while we have

$$
M_{b f}(t)=\left(t_{1}+t_{2}, t_{1}+t_{2}+t_{3}\right)=t_{1}(1,1)+t_{2}(1,1)+t_{3}(0,1)+t_{4}(0,0)+t_{5}(0,0)
$$

and

$$
M_{f}(t)=\left(\begin{array}{cc}
0 & t_{1}+t_{2} \\
-\left(t_{1}+t_{2}\right) & 0
\end{array}\right)
$$

Thus, we obtain in this case

$$
\begin{gathered}
M_{b f}(t) M_{f}(t)^{-1} M_{f b}(t)=\left(t_{1}+t_{2}, t_{1}+t_{2}+t_{3}\right)\left(\begin{array}{cc}
0 & \frac{-1}{t_{1}+t_{2}} \\
\frac{1}{t_{1}+t_{2}} & 0
\end{array}\right)\left(\begin{array}{c}
t_{1}+t_{2} \\
t_{1}+t_{2}+t_{3}
\end{array}\right) \\
=\left(t_{1}+t_{2}, t_{1}+t_{2}+t_{3}\right)\left(\begin{array}{c}
\frac{-\left(t_{1}+t_{2}+t_{3}\right)}{t_{1}+t_{2}} \\
1
\end{array}\right)=-\left(t_{1}+t_{2}+t_{3}\right)+t_{1}+t_{2}+t_{3} \equiv 0 .
\end{gathered}
$$

Thus, in this particular example we have $M_{b f}(t) M_{f}(t)^{-1} M_{f b}(t) \equiv 0$ for all $t=\left(t_{1}, \ldots, t_{5}\right)$, so that $\operatorname{Ber}(\mathcal{M}(t))=\operatorname{det}\left(M_{b}(t)\right) \operatorname{det}\left(M_{f}(t)\right)^{-1}=\left(t_{1}+t_{2}+t_{3}\right) /\left(t_{1}+t_{2}\right)^{2}$ and the locus of zeros and poles $\mathcal{X}_{(\Gamma, B)} \subset \mathbb{P}^{5 \mid 8}$ is the union of $t_{1}+t_{2}+t_{3}=0$ and $t_{1}+t_{2}=0$ in $\mathbb{P}^{5}$ (the latter counted with multiplicity two), with the restriction of the sheaf from $\mathbb{P}^{5 / 8}$.

3.6. The universality property. Lemma 3.9 below shows to what extent the "universality property" of graph hypersurfaces, i.e. the fact that they generate the Grothendieck group of varieties, continues to hold when passing to supermanifolds.

Lemma 3.9. Let $\mathcal{R}$ be the subring of the Grothendieck ring $K_{0}\left(\mathcal{S} \mathcal{V}_{\mathbb{C}}\right)$ of supermanifolds spanned by the $\left[\mathcal{X}_{(\Gamma, B)}\right]$, for $\mathcal{X}_{(\Gamma, B)}$ the graph supermanifolds defined by the divisor of zeros and poles of the Berezinian $\operatorname{Ber}(\mathcal{M}(t))$, with $B$ a choice of a basis of $H_{1}(\Gamma)$. Then

$$
\mathcal{R}=K_{0}\left(\mathcal{V}_{\mathbb{C}}\right)\left[T^{2}\right] \subset K_{0}\left(\mathcal{S} \mathcal{V}_{\mathbb{C}}\right)
$$

where $T=\left[\mathbb{A}^{0 \mid 1}\right]$.

Proof. By Corollary 2.4 and the universality result of [3], it suffices to prove that the subring of $K_{0}\left(\mathcal{S} \mathcal{V}_{\mathbb{C}}\right)$ generated by the $\left[\mathcal{X}_{(\Gamma, B)}\right]$ contains the classes of the ordinary graph hypersurfaces in $K_{0}\left(\mathcal{V}_{\mathbb{C}}\right)$ and the class $\left[\mathbb{A}^{0 \mid 2}\right]$. 


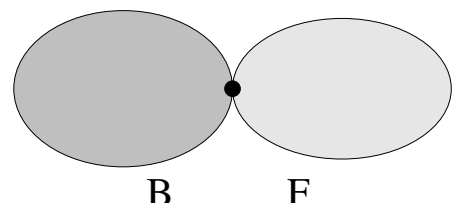

FiguRE 4. Graphs with $\ell_{b f}=0$.

To show that $\mathcal{R}$ contains the ordinary graph hypersurfaces, consider the special class of graphs that are of the form schematically illustrated in Figure 4. These are unions of two graphs, one only with bosonic edges and one only with fermionic edges, with a single vertex in common. Notice that in actual physical theories the combinatorics of graphs with only fermionic edges is severely restricted (see $20, \S 5.3$ ) depending on the dimension $D$ in which the theory is considered. However, for the purpose of this universality result, we allow arbitrary $D$ and corresponding graphs, just as in the result of 3 . one does not restrict to the Feynman graphs of any particular theory.

The graphs of Figure 4 provide examples of graphs with bases of $H_{1}(\Gamma)$ containing loops with only fermionic or only bosonic legs, i.e. with $\ell_{b f}=0, \ell_{f}=\ell_{f f}$ and $\ell_{b}=\ell_{b b}$. This implies that, for all these graphs $\Gamma=\Gamma_{B} \cup_{v} \Gamma_{F}$ with the corresponding bases of $H_{1}$, one has $M_{b f}(t) \equiv 0$, since for each edge variable $t_{i}$ one of the two factors $\eta_{i r_{b}} \eta_{i r_{f}}$ is zero. Thus, for this class of examples we have $\operatorname{Ber}(\mathcal{M}(t))=\operatorname{det}\left(M_{b}(t)\right) / \operatorname{det}\left(M_{f}(t)\right)$. Moreover, we see that for these examples $\operatorname{det}\left(M_{b}(t)\right)=\Psi_{\Gamma_{b}}(t)$ is the usual graph polynomial of the graph $\Gamma_{B}$ with only bosonic edges. Since such $\Gamma_{B}$ can be any arbitrary ordinary graph, we see that the locus of zeros alone, and just for this special subset of the possible graphs, already suffices to generate the full $K_{0}\left(\mathcal{V}_{\mathbb{C}}\right)$ since it gives all the graph varieties $\left[X_{\Gamma_{B}}\right]$.

To show then that the subring $\mathcal{R}$ contains the classes $\left[\mathbb{A}^{0 \mid 2 f}\right]$, for all $f$, first notice that the classes $\left[\mathbb{P}^{n}\right]\left[\mathbb{A}^{0 \mid 2 f}\right]=[p t]\left[\mathbb{A}^{0 \mid 2 f}\right]+\left[\mathbb{A}^{1 \mid 0}\right]\left[\mathbb{A}^{0 \mid 2 f}\right]+\cdots+\left[\mathbb{A}^{n \mid 0}\right]\left[\mathbb{A}^{0 \mid 2 f}\right]$ belong to $\mathcal{R}$, for all $n$ and $f$. These are supplied, for instance, by the graphs with a single loop containing fermionic edges, as observed above. This implies that elements of the form $\left[\mathbb{A}^{n \mid 0}\right]\left[\mathbb{A}^{0 \mid 2 f}\right]=\left[\mathbb{P}^{n}\right]\left[\mathbb{A}^{0 \mid 2 f}\right]-\left[\mathbb{P}^{n-1}\right]\left[\mathbb{A}^{0 \mid 2 f}\right]$ belong to $\mathcal{R}$. In particular the graph consisting of a single fermionic edge closed in a loop gives $\left[\mathbb{A}^{0 \mid 2 f}\right]$ in $\mathcal{R}$.

Notice that in [3], in order to prove that the correspoding graph hypersurfaces generate $K_{0}\left(\mathcal{V}_{\mathbb{C}}\right)$, one considers all graphs and not only the log divergent ones with $n=D \ell / 2$, even though only for the log divergent ones the period has the physical interpretation as Feynman integral. Similarly, here, in Lemma 3.9, we consider all $(\Gamma, B)$ and not just those satisfying the condition (3.42).

The fact that we only find classes of the even dimensional superplanes $\left[\mathbb{A}^{0 \mid 2 f}\right]$ in $\mathcal{R}$ instead of all the possible classes $\left[\mathbb{A}^{0 \mid f}\right]$ is a consequence of the fermion doubling used in Lemma 3.4 in the representation of the Feynman integral in terms of an ordinary and a fermionic integration.

\section{SUPERMANIFOLDS AND MIRRORS}

We discuss here some points of contact between the construction we outlined in this paper and the supermanifolds and periods that appear in the theory of mirror symmetry.

Supermanifolds arise in the theory of mirror symmetry (see for instance [23, 2], 13]) in order to describe mirrors of rigid Calabi-Yau manifolds, where the lack of moduli of complex structures prevents the existence of Kähler moduli on the mirror. The mirror still exists, not as a conventional Kähler manifold, but as a supermanifold embedded in a (weighted) super-projective space. 
For instance, in the construction given in [23], one considers the hypersurface in (weighted) projective space given by the vanishing of a superpotential $X=\{W=0\} \subset \mathbb{P}^{n}$. The local ring of the hypersurface $X$ is given by polynomials in the coordinates modulo the Jacobian ideal $\mathcal{R}_{X}=\mathbb{C}\left[x_{i}\right] / d W\left(x_{i}\right)$. To ensure the vanishing of the first Chern class, one corrects the superpotential $W$ by additional quadratic terms in either bosonic or fermionic variables, so that the condition $W=0$ defines a supermanifold embedded in a (weighted) super-projective space, instead of an ordinary hypersurface in projective space.

In the ordinary case, one obtains the primitive part of the middle cohomology $H_{0}^{n-1}(X)$ and its Hodge decomposition via the Poincaré residue

$$
\operatorname{Res}(\omega)=\int_{C} \omega
$$

with $C$ a 1-cycle encircling the hypersurface $X$, applied to forms of the form

$$
\omega(P)=\frac{P\left(x_{0}, \ldots, x_{n}\right) \Omega}{W^{k}},
$$

with $\Omega=\sum_{i=0}^{n}(-1)^{i} \lambda_{i} x_{i} d x_{0} \cdots \widehat{d x_{i}} \cdots d x_{n}$, as in (1.3) with $\lambda_{i}$ the weights in the case of weighted projective spaces, and with $P \in \mathcal{R}_{X}$ satisfying $k \operatorname{deg}(W)=\operatorname{deg}(P)+\sum_{i} \lambda_{i}$.

In the supermanifold case, one replaces the calulation of the Hodge structure on the mirror done using the technique described above, by a supergeometry analog, where the forms (4.2) are replaced by forms

$$
\frac{P\left(x_{0}, \ldots, x_{n}\right) d \theta_{1} \cdots d \theta_{2 m} \Omega}{W^{k}}
$$

where here the superpotential $W$ is modified by the presence of an additional quadratic term in the fermionic variables $\theta_{1} \theta_{2}+\cdots \theta_{2 m-1} \theta_{2 m}$.

In comparison to the setting discussed in this paper, notice that the procedure of replacing the potential $W$ by $W^{\prime}=W+\theta_{1} \theta_{2}+\cdots \theta_{2 m-1} \theta_{2 m}$, with the additional fermionic integration, is very similar to the first step in our derivation where we replaced the original expression $T=t_{1} q_{1}+\cdots t_{n} q_{n}$ by the modified one $T+\frac{1}{2} \theta^{\tau} \mathcal{Q} \theta$ with $\frac{1}{2} \theta^{\tau} \mathcal{Q} \theta=\mathrm{q}_{1} \theta_{1} \theta_{2}+$ $\cdots+\mathrm{q}_{f} \theta_{2 f-1} \theta_{2 f}$. Thus, replacing the ordinary integration $\int T^{-n}(t) d t$ by the integration $\int\left(T(t)+\frac{1}{2} \theta^{\tau} \mathcal{Q} \theta\right)^{-n+f} d t d \theta$ is an analog of replacing the integral $\int W^{-k} d t$ with the integral $\int\left(W+\theta_{1} \theta_{2}+\cdots \theta_{2 m-1} \theta_{2 m}\right)^{-k} d t d \theta$ used in the mirror symmetry context. However, there seems to be no analog, in that setting, for the type of periods of the form (3.45) that we obtain here and for the corresponding type of supermanifolds defined by divisors of Berezinians considered here.

\section{REFERENCES}

[1] D. Abramovich, K. Karu, K. Matsuki, J. Wlodarczyk, Torification and factorization of birational maps, J. Amer. Math. Soc., Vol.15 (2002), N.3, 531-572.

[2] M. Aganagic, C. Vafa, Mirror symmetry and supermanifold, Adv. Theor. Math. Phys., Vol.8 (2004) 939-954.

[3] P. Belkale, P. Brosnan, Matroids, motives, and a conjecture of Kontsevich, Duke Math. Journal, Vol.116 (2003) 147-188.

[4] C. Bergbauer, A. Rej, Insertion of graphs and singularities of graph hypersurfaces, preprint.

[5] I.N. Bernšteĭn, D.A. Leı̆tes, Integral forms and the Stokes formula on supermanifolds. Functional Anal. Appl. 11 (1977), no. 1, 45-47.

[6] J. Bjorken, S. Drell, Relativistic Quantum Mechanics, McGraw-Hill, 1964, and Relativistic Quantum Fields, McGraw-Hill, 1965.

[7] S. Bloch, Motives associated to graphs, Japan J. Math., Vol.2 (2007) 165-196.

[8] S. Bloch, E. Esnault, D. Kreimer, On motives associated to graph polynomials, Commun. Math. Phys., Vol.267 (2006) 181-225.

[9] D. Broadhurst, D. Kreimer, Association of multiple zeta values with positive knots via Feynman diagarams up to 9 loops, Phys. Lett. B, Vol.393 (1997) 403-412. 
[10] A. Connes, D. Kreimer, Renormalization in quantum field theory and the Riemann-Hilbert problem I. The Hopf algebra structure of graphs and the main theorem, Comm. Math. Phys., Vol.210 (2000) $249-273$.

[11] A. Connes, M. Marcolli, Renormalization and motivic Galois theory. International Math. Res. Notices (2004) N.76, 4073-4091.

[12] M. Kashiwara, P. Shapira, Sheaves on manifolds, Springer, 1990.

[13] S.P. Kumar, G. Policastro, Strings in twistor superspace and mirror symmetry, Phys. Lett. B, Vol.619 (2005) 163-170.

[14] M. Larsen, V. Lunts, Motivic measures and stable birational geometry, Moscow Math. Journal, Vol.3 (2003), N.1, 85-95.

[15] C. LeBrun, Y.S. Poon, R.O. Wells, Projective embeddings of complex supermanifolds, Commun. Math. Phys., Vol.126 (1990) 433-452.

[16] Yu.I. Manin, Gauge field theory and complex geometry, Springer Verlag, 1988.

[17] P.B. Medvedev, $\alpha$ representation in scalar supersymmetric theory, Teoreticheskaya i matematicheskaya fizika (transl.Institute of Theoretical and Experimental Physics) Vol.35 (1978) N.1, 37-47.

[18] N. Nakanishi Graph Theory and Feynman Integrals. Gordon and Breach, 1971.

[19] I.B. Penkov, I.A. Skornyakov, Projectivity and D-affinity of flag supermanifolds, Russ. Math. Surv., Vol.40 (1987) 233-234.

[20] P. Ramond, Field theory: a modern primer. Addison Wesley, 1990.

[21] A.A. Rosly, A.S. Schwarz, A.A. Voronov, Geometry of superconformal manifolds, Comm. Math. Phys., Vol.119 (1988) 129-152.

[22] A. Schwarz, I. Shapiro, Supergeometry and arithmetic geometry. Nuclear Phys. B 756 (2006), N.3, 207-218.

[23] S. Sethi, Supermanifolds, rigid manifolds and mirror symmetry. Nuclear Phys. B 430 (1994), no. 1, $31-50$.

[24] W. van Suijlekom, The Hopf algebra of Feynman graphs in quantum electrodynamics. Lett. Math. Phys. 77 (2006), N.3, 265-281.

Max-Planck Institut für Mathematik, Vivatsgasse 7, Bonn, D-53111 Germany

E-mail address: marcolli@mpim-bonn.mpg.de

E-mail address: rej@mpim-bonn.mpg.de 